\title{
Analysis of Third-Grade Fluid in Helical Screw Rheometer
}

\author{
M. Zeb, ${ }^{1}$ S. Islam, ${ }^{2}$ A. M. Siddiqui, ${ }^{3}$ and T. Haroon ${ }^{1}$ \\ ${ }^{1}$ Department of Mathematics, COMSATS Institute of Information Technology, Islamabad, Pakistan \\ ${ }^{2}$ Department of Mathematics, Abdul Wali Khan University, Mardan, Pakistan \\ ${ }^{3}$ Department of Mathematics, Pennsylvania State University, York Campus, York, PA 17 403, USA
}

Correspondence should be addressed to M. Zeb; immzeb@yahoo.com

Received 17 October 2012; Revised 4 March 2013; Accepted 20 March 2013

Academic Editor: Juan Torregrosa

Copyright (c) 2013 M. Zeb et al. This is an open access article distributed under the Creative Commons Attribution License, which permits unrestricted use, distribution, and reproduction in any medium, provided the original work is properly cited.

\begin{abstract}
The steady flow of an incompressible, third-grade fluid in helical screw rheometer (HSR) is studied by "unwrapping or flattening" the channel, lands, and the outside rotating barrel. The geometry is approximated as a shallow infinite channel, by assuming that the width of the channel is large as compared to the depth. The developed second-order nonlinear coupled differential equations are reduced to single differential equation by using a transformation. Using Adomian decomposition method, analytical expressions are calculated for the the velocity profiles and volume flow rates. The results have been discussed with the help of graphs as well. We observed that the velocity profiles are strongly dependant on non-Newtonian parameter $(\widetilde{\beta})$, and with the increase in $\widetilde{\beta}$, the velocity profiles increase progressively, which conclude that extrusion process increases with the increase in $\widetilde{\beta}$. We also observed that the increase in pressure gradients in $x$ - and $z$-direction increases the net flow inside the helical screw rheometer, which increases the extrusion process. We noticed that the flow increases as the flight angle increase.
\end{abstract}

\section{Introduction}

In real life, there are many materials that exhibit the mechanical characteristics of both elasticity and viscosity. These materials are known as non-Newtonian fluids. These fluids cannot be described satisfactorily by the theory of elasticity or viscosity but by a combination of both. Due to the rheological behavior of these fluids, many constitutive equations are proposed [1]. In most fluid food products, the shear stress is dependent on the share rate; hence, nonlinear flow curve results and a unique viscosity are no longer adequate to characterize the fluid. Many fluids such as molten plastics, polymers, and slurries are non-Newtonian in their flow behavior. The basic governing equations for such fluids motion are highly nonlinear differential equations having no general solution, and only a limited number of exact solutions have been established for particular problems. To solve practical problems in engineering and mathematics, researchers and scientists have developed numerous numerical techniques, that is, finite difference method (FDM), finite volume approach, control-volume-based finite element method (CVFEM), lattice Boltzmann method (LBM), and analytical techniques, that is, variational iteration method (VIM), perturbation method (PM), homotopy perturbation method (HPM), HPM-Pade technique, homotopy analysis method (HAM), optimal homotopy analysis method (OHAM), optimal homotopy perturbation method (OHPM), and some other techniques, to overcome nonlinearity and get numerical and analytical solutions [2-10]. A brief review on analytical techniques is presented by [11]. In recent years in the area of series solutions, an iterative technique Adomian decomposition method $[12,13]$ has received much attention. A considerable amount of research work has been invested in the application of this method to a wide class of linear, nonlinear, and partial differential equations and integral equations. Many interesting problems in applied science and engineering have been successfully solved by using ADM to their higher degree of accuracy. A useful quality of the $\mathrm{ADM}$ is that it has proved to be a competitive alternative to the Taylor series method and other series techniques. This method has been used in obtaining analytic and approximate solutions to a wide class of linear and nonlinear, differential and integral equations, homogeneous or inhomogeneous, with constant coefficients or with variable coefficients. 
The Adomian decomposition method is comparatively easier to program in engineering problems than other series methods and provides immediate and visible solution terms without linearization, perturbation, or discretization of the problem, while the physical behavior of the solution remains unchanged. It provides analytical solution in the form of an infinite series in which each term can be easily determined [14-16]. If an exact solution exists for the problem, then the obtained series converges very rapidly to the solution. For concrete problems, where a closed-form solution is not obtainable, a truncated number of terms are usually used for numerical purposes [17].

The Helical Screw Rheometer (HSR) consists of a helical screw in a tight fitting cylinder, with the inlet and outlet parts closing the inner screw. Rotation of screw creates a pressure gradient along the axis of the screw. The HSR is being used for rheological measurements of fluid food suspensions. The geometry of an HSR is similar to a single-screw extruder [18]. Extrusion process is widely used in multigrade oils, liquid detergents, paints, polymer solutions and polymer melts [19], the injection molding process for polymeric materials, the production of pharmaceutical products, food extrusion, and processing of plastics [20]. Various food items in daily life, such as cookie dough, sevai, pastas, breakfast cereals, french fries, baby food, ready to eat snacks, and dry pet food, are most commonly manufactured using the extrusion process.

Knowledge of rheological properties is essential in the processing of fluid foods since these affect the flow behavior. During processing, physical and chemical changes can occur so it is desirable to monitor the process to achieve excellent output and quality control [21]. On-line rheological measurements in the food industry have been limited [22].

Bird et al. [23] presented an asymptotic solution and arbitrary values of the flow behavior index, for the powerlaw fluid in a very thin annulus. A brief discussion is given by Mohr and Mallouk [24] for the same problem considering Newtonian fluid in a screw extruder. Tamura et al. [18] also investigated the flow of Newtonian fluid in helical screw rheometer.

The objective of this paper is to study the flow of thirdgrade fluid in helical screw rheometer (HSR) where the effects of curvature and also of flights are neglected by assuming that the helical channel is "unwrapped." The geometry is approximated as a shallow infinite channel, with $h / B \ll 1$, where $B$ denotes the channel width and $h$ is gap [18]. The formulation results in second-order nonlinear coupled differential equations which are reduced to first-order nonlinear differential equations by integrating and combined in single first-order differential equation using a transformation, the solution is obtained by using ADM. Analytical expressions are given for the velocity components in $x$-, $z$-directions and in direction of the screw axis. Volume flow rates are also obtained for all three types of velocities. The paper is organized as follows. Section 2 contains the governing equations of the fluid model. In Section 3, the problem under consideration is formulated. In Section 4, description of Adomian decomposition method is given. In Section 5, the governing equation of the problem is solved. In Section 6, results are discussed. Section 7 contains conclusion.

\section{Basic Equations}

The basic equations governing the motion of an incompressible fluid are

$$
\begin{gathered}
\operatorname{div} \mathbf{V}=0, \\
\rho \frac{D \mathbf{V}}{D t}=\rho \mathbf{f}+\operatorname{div} \mathbf{T},
\end{gathered}
$$

where $\rho$ is the constant fluid density, $\mathbf{V}$ is the velocity vector, $\mathbf{f}$ is the body force per unit mass, the $D / D t$ denotes the material time derivative defined as

$$
\frac{D(*)}{D t}=\frac{\partial}{\partial t}(*)+(\mathbf{V} \cdot \nabla)(*),
$$

and $\mathbf{T}$ is the Cauchy stress tensor, given as

$$
\mathbf{T}=-P \mathbf{I}+\mathbf{S},
$$

where $P$ denotes the dynamic pressure, I denotes unit tensor, and $\mathbf{S}$ denotes the extra stress tensor. The constitutive equation for third-grade fluid is defined as

$$
\begin{aligned}
\mathbf{S}= & \mu \mathbf{A}_{1}+\alpha_{1} \mathbf{A}_{2}+\alpha_{2} \mathbf{A}_{1}^{2}+\beta_{1} \mathbf{A}_{3} \\
& +\beta_{2}\left(\mathbf{A}_{1} \mathbf{A}_{2}+\mathbf{A}_{2} \mathbf{A}_{1}\right)+\beta_{3}\left(\operatorname{tr} \mathbf{A}_{1}^{2}\right) \mathbf{A}_{1},
\end{aligned}
$$

where $\mu$ is the viscosity, $\alpha_{1}, \alpha_{2}, \beta_{1}, \beta_{2}$, and $\beta_{3}$ are the material constants, $\mathbf{A}_{1}, \mathbf{A}_{2}$, and $\mathbf{A}_{3}$ are the first three Rivlin-Ericksen tensors defined as [19]

$$
\begin{gathered}
\mathbf{A}_{1}=(\operatorname{grad} \mathbf{V})+(\operatorname{grad} \mathbf{V})^{T}, \\
\mathbf{A}_{n+1}=\frac{D \mathbf{A}_{n}}{D t}+\left[\mathbf{A}_{n}(\operatorname{grad} \mathbf{V})+(\operatorname{grad} \mathbf{V})^{T} \mathbf{A}_{n}\right], \quad(n=1,2)
\end{gathered}
$$

\section{Problem Formulation}

Consider the steady flow of an isothermal, incompressible and homogeneous third-grade fluid in helical screw rheometer (HSR) in such a way that the curvature of the screw channel is ignored, unrolled and laid out on a flat surface. The barrel surface is also flattened. Assume that the screw surface, the lower plate, is stationary, and the barrel surface, the upper plate, is moving across the top of the channel with velocity $V$ at an angle $\phi$ to the direction of the channel Figure 1. The phenomena is the same as the barrel held stationary and the screw rotates. The geometry is approximated as a shallow infinite channel, by assuming that the width $B$ of the channel is large compared with the depth $h$; edge effects in the fluid at the land are ignored. The coordinate axes are positioned in such a way that the $x$-axis is perpendicular to the wall and $z$-axis is in down channel direction. The liquid wets all the surfaces and moves by the shear stresses produced by the relative movement of the barrel and channel. For simplicity, the velocity of the barrel relative to the channel is broken up into two components: $U$ is along $x$-axis and $W$ is along $z$-axis 

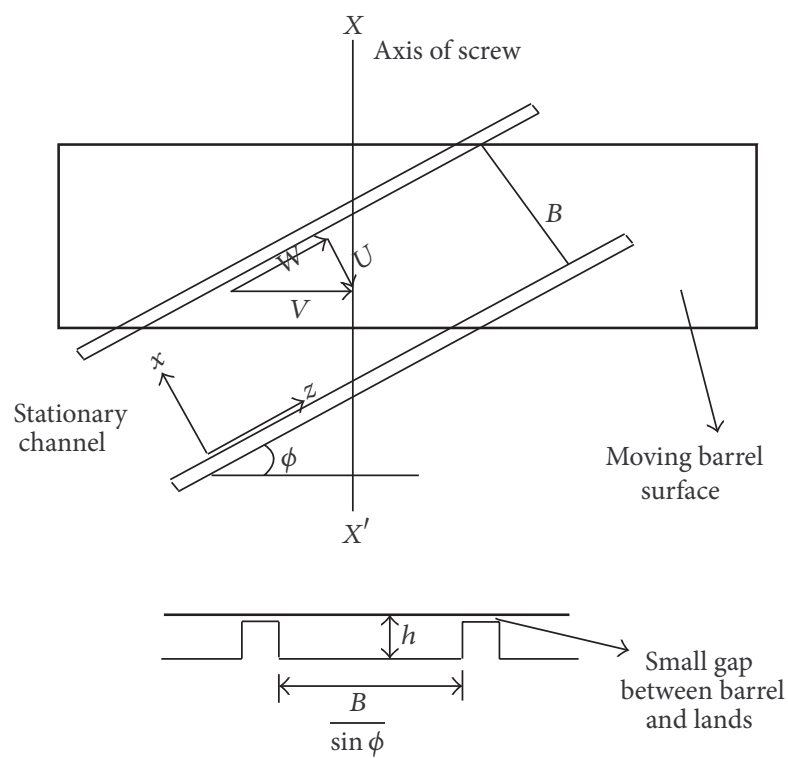

Figure 1: The geometry of the "unwrapped" screw channel and barrel surface.

[24]. Under these assumptions the velocity field and cauchy stress tensor can be written as

$$
\mathbf{V}=[u(y), 0, w(y)], \quad \mathbf{T}=\mathbf{T}(y) .
$$

On substituting (7) in (4) and (5), we obtain nonzero components of Cauchy stress $\mathbf{T}$,

$$
\begin{gathered}
\tau_{x x}=-P+\alpha_{2}\left(\frac{d u}{d y}\right)^{2}, \\
\tau_{x y}=\tau_{y x}=\mu \frac{d u}{d y}+2\left(\beta_{2}+\beta_{3}\right)\left\{\left(\frac{d u}{d y}\right)^{2}+\left(\frac{d w}{d y}\right)^{2}\right\} \frac{d u}{d y} \\
\tau_{x z}=\tau_{z x}=\alpha_{2} \frac{d u}{d y} \frac{d w}{d y}, \\
\tau_{y y}=-P+\left(2 \alpha_{1}+\alpha_{2}\right)\left[\left(\frac{d u}{d y}\right)^{2}+\left(\frac{d w}{d y}\right)^{2}\right] \\
\tau_{y z}=\tau_{z y}=\mu \frac{d w}{d y}+2\left(\beta_{2}+\beta_{3}\right)\left\{\left(\frac{d u}{d y}\right)^{2}+\left(\frac{d w}{d y}\right)^{2}\right\} \frac{d w}{d y} \\
\tau_{z z}=-P+\alpha_{2}\left(\frac{d w}{d y}\right)^{2},
\end{gathered}
$$

Using (7), (1) is identically satisfied, and (2) in the absence of body forces results in

$$
\begin{aligned}
0= & -\frac{\partial P}{\partial x}+\frac{\partial}{\partial y} \\
& \times\left[\mu \frac{d u}{d y}+2\left(\beta_{2}+\beta_{3}\right)\left\{\left(\frac{d u}{d y}\right)^{2}+\left(\frac{d w}{d y}\right)^{2}\right\} \frac{d u}{d y}\right], \\
0= & -\frac{\partial P}{\partial y}+\frac{1}{2}\left(2 \alpha_{1}+\alpha_{2}\right) \frac{\partial}{\partial y}\left[\left(\frac{d u}{d y}\right)^{2}+\left(\frac{d w}{d y}\right)^{2}\right], \\
0= & \frac{\partial P}{\partial z}+\frac{\partial}{\partial y} \\
& \times\left[\mu \frac{d w}{d y}+2\left(\beta_{2}+\beta_{3}\right)\left\{\left(\frac{d u}{d y}\right)^{2}+\left(\frac{d w}{d y}\right)^{2}\right\} \frac{d w}{d y}\right] .
\end{aligned}
$$

Define the modified pressure $\widehat{P}$ as

$$
\widehat{P}=P-\left(\alpha_{1}+\frac{1}{2} \alpha_{2}\right)\left\{\left(\frac{d u}{d y}\right)^{2}+\left(\frac{d w}{d y}\right)^{2}\right\},
$$

which implies that $\widehat{P}=\widehat{P}(x, z)$ only; thus, (9) reduce to

$$
\begin{aligned}
& \frac{d^{2} u}{d y^{2}}+\frac{2\left(\beta_{2}+\beta_{3}\right)}{\mu} \frac{d}{d y}\left[\left\{\left(\frac{d u}{d y}\right)^{2}+\left(\frac{d w}{d y}\right)^{2}\right\} \frac{d u}{d y}\right]=\frac{1}{\mu} \frac{\partial \widehat{P}}{\partial x}, \\
& \frac{d^{2} w}{d y^{2}}+\frac{2\left(\beta_{2}+\beta_{3}\right)}{\mu} \frac{d}{d y}\left[\left\{\left(\frac{d u}{d y}\right)^{2}+\left(\frac{d w}{d y}\right)^{2}\right\} \frac{d w}{d y}\right]=\frac{1}{\mu} \frac{\partial \widehat{P}}{\partial z} .
\end{aligned}
$$

The associated boundary conditions can be taken as (see Figure 1)

$$
\begin{aligned}
& u=0, \quad w=0, \quad \text { at } y=0, \\
& u=U, \quad w=W, \quad \text { at } y=h,
\end{aligned}
$$

where

$$
U=-V \sin \phi, \quad W=V \cos \phi .
$$

Introducing nondimensionalized parameters

$$
\begin{gathered}
x^{*}=\frac{x}{h}, \quad y^{*}=\frac{y}{h}, \quad z^{*}=\frac{z}{h}, \quad u^{*}=\frac{u}{W}, \quad w^{*}=\frac{w}{W}, \\
P^{*}=\frac{P}{\mu(W / h)}, \quad \tilde{\beta}^{*}=\frac{\left(\beta_{2}+\beta_{3}\right) W^{2}}{\mu h^{2}},
\end{gathered}
$$

where $\mathbf{T}=\left[\tau_{i j}\right]$. 
in (11)-(12), takes the form

$$
\begin{aligned}
\frac{d^{2} u^{*}}{d y^{* 2}}+\widetilde{\beta}^{*} \frac{d}{d y^{*}}\left[\left\{\left(\frac{d u^{*}}{d y^{*}}\right)^{2}+\left(\frac{d w^{*}}{d y^{*}}\right)^{2}\right\} \frac{d u^{*}}{d y^{*}}\right]=\frac{\partial P^{*}}{\partial x^{*}}, \\
\frac{d^{2} w^{*}}{d y^{* 2}}+\widetilde{\beta}^{*} \frac{d^{*}}{d y^{*}}\left[\left\{\left(\frac{d u^{*}}{d y^{*}}\right)^{2}+\left(\frac{d w^{*}}{d y^{*}}\right)^{2}\right\} \frac{d w^{*}}{d y^{*}}\right]=\frac{\partial P^{*}}{\partial z^{*}} \\
u^{*}=0, \quad w^{*}=0, \quad \text { at } y^{*}=0, \\
u^{*}=\frac{U}{W}, \quad w^{*}=1, \quad \text { at } y^{*}=1 .
\end{aligned}
$$

Dropping "*" from (15) onward and defining

$$
F=u+\imath w, \quad V_{0}=\frac{U}{W}+\iota 1, \quad G=P_{, x}+\iota P_{, z},
$$

where $\partial P / \partial x=P_{, x}, \partial P / \partial z=P_{, z}$ in (15) reduce to

$$
\frac{d^{2} F}{d y^{2}}=G-\tilde{\beta}\left\{\left(\frac{d F}{d y}\right)^{2} \frac{d^{2} \bar{F}}{d y^{2}}+2 \frac{d F}{d y} \frac{d^{2} F}{d y^{2}} \frac{d \bar{F}}{d y}\right\},
$$

where $\bar{F}$ is the complex conjugate of $F$.

The boundary conditions become

$$
\begin{aligned}
& F=0 \quad \text { at } y=0, \\
& F=V_{0} \quad \text { at } y=1 .
\end{aligned}
$$

Equation (17) is second-order nonlinear ordinary differential equation, and the exact solution seems to be difficult. In the following section, we use Adomian decomposition method to obtain the approximate solution. To obtain the expressions for the velocity components in $x$ - and $z$-directions, (17) together with the boundary conditions (18) is solved up to the second component approximations by using the symbolic computation software Wolfram Mathematica 7.

\section{Description of Adomian Decomposition Method}

Consider equation $J[F(y)]=g(y)$, where $J$ represents a general nonlinear ordinary or partial differential operator including both linear and nonlinear terms. The linear terms are decomposed into $L+R$, where $L$ is invertible. $L$ is taken as the highest-order derivative to avoid difficult integrations, and $R$ is the remainder of the linear operator. Thus, the equation can be written as

$$
L(F)+R(F)+N(F)=g(y),
$$

where $N(F)$ indicates the nonlinear term and $g(y)$ is forcing function. Since $L$ is invertible, so $L^{-1}$ exist. The above equation can be written as

$$
L^{-1} L(F)=L^{-1} g(y)-L^{-1} R(F)-L^{-1} N(F) .
$$

If $L\left(=d^{2} / d y^{2}\right)$ is a second-order operator, $L^{-1}(=$ $\left.\iint(*) d y d y\right)$ is a twofold indefinite integral. Equation (20) becomes

$$
F=C_{1}+C_{2} y+L^{-1} g(y)-L^{-1} R(F)-L^{-1} N(F),
$$

where $C_{1}$ and $C_{2}$ are constants of integration and can be determined by using boundary or initial conditions. ADM assumes that the solution $F$ can be expanded into infinite series as $F=\sum_{n=0}^{\infty} F_{n}$; also, the nonlinear term $N(F)$ will be written as $N(F)=\sum_{n=0}^{\infty} A_{n}$, where $A_{n}$ are special Adomian polynomials which can be defined as

$$
A_{n}=\frac{1}{n !}\left[\frac{d^{n}}{d \lambda^{n}}\left\{N\left(\sum_{i=0}^{\infty} \lambda^{i} F_{i}\right)\right\}\right]_{\lambda=0} n=0,1,2,3, \ldots,
$$

finally, the solution can be written as

$$
\sum_{n=0}^{\infty} F_{n}=F_{0}-L^{-1} R\left(\sum_{n=0}^{\infty} F_{n}\right)-L^{-1}\left(\sum_{n=0}^{\infty} A_{n}\right),
$$

where

$$
F_{0}=C_{1}+C_{2} y+L^{-1} g(y)
$$

is initial solution and

$$
F_{n}=-L^{-1} R\left(F_{n-1}\right)-L^{-1} A_{n-1}, \quad n \geq 1,
$$

and (25) is $n$ th-order solution. The practical solution will be the $n$-term approximation

$$
\phi_{n}=\sum_{i=0}^{n-1} F_{i}
$$

and by definition [25-27],

$$
\lim _{n \rightarrow \infty} \phi_{n}=\sum_{i=0}^{n-1} F_{i}=F
$$

\section{Solution of the Problem}

Adomian decomposition method describes that in the operator form (17) can be written as

$$
L(F)=G-\widetilde{\beta}\left\{\left(\frac{d F}{d y}\right)^{2} \frac{d^{2} \bar{F}}{d y^{2}}+2 \frac{d F}{d y} \frac{d^{2} F}{d y^{2}} \frac{d \bar{F}}{d y}\right\},
$$

where $L$ is the differential operator taken as the highest-order derivative to avoid difficult integrations, assuming that $L$ is invertible, which implies that $L^{-1}=\iint(*) d y d y$ exist.

On applying $L^{-1}$ to both sides of (28) results in

$$
\begin{aligned}
F= & A+B y+L^{-1}(G)-\widetilde{\beta} L^{-1} \\
& \times\left\{\left(\frac{d F}{d y}\right)^{2} \frac{d^{2} \bar{F}}{d y^{2}}+2 \frac{d F}{d y} \frac{d^{2} F}{d y^{2}} \frac{d \bar{F}}{d y}\right\},
\end{aligned}
$$


where $A$ and $B$ are constants of integration and can be determined by using boundary conditions. According to procedure of Adomian decomposition method $F$ and $\bar{F}$ can be written in component form as:

$$
\begin{aligned}
& F=\sum_{n=0}^{\infty} F_{n}, \\
& \bar{F}=\sum_{n=0}^{\infty} \bar{F}_{n} .
\end{aligned}
$$

Thus, (29) takes the form

$$
\begin{aligned}
\sum_{n=0}^{\infty} F_{n}=A+ & B y+L^{-1}(G)-\widetilde{\beta} L^{-1} \\
\times & \left\{\left(\frac{d}{d y}\left(\sum_{n=0}^{\infty} F_{n}\right)\right)^{2}\left(\frac{d^{2}}{d y^{2}}\left(\sum_{n=0}^{\infty} \bar{F}_{n}\right)\right)\right. \\
& +2\left(\frac{d}{d y}\left(\sum_{n=0}^{\infty} F_{n}\right)\right)\left(\frac{d^{2}}{d y^{2}}\left(\sum_{n=0}^{\infty} F_{n}\right)\right) \\
& \left.\times\left(\frac{d}{d y}\left(\sum_{n=0}^{\infty} \bar{F}_{n}\right)\right)\right\} .
\end{aligned}
$$

Adomian also suggested that the nonlinear terms can be explored in the form of Adomian polynomials, say, $A_{n}$ and $B_{n}$ as

$$
\begin{aligned}
& \sum_{n=0}^{\infty} A_{n}=\left(\frac{d}{d y}\left(\sum_{n=0}^{\infty} F_{n}\right)\right)^{2}\left(\frac{d^{2}}{d y^{2}}\left(\sum_{n=0}^{\infty} \bar{F}_{n}\right)\right), \\
& \sum_{n=0}^{\infty} B_{n}=2\left(\frac{d}{d y}\left(\sum_{n=0}^{\infty} F_{n}\right)\right)\left(\frac{d^{2}}{d y^{2}}\left(\sum_{n=0}^{\infty} F_{n}\right)\right)\left(\frac{d}{d y}\left(\sum_{n=0}^{\infty} \bar{F}_{n}\right)\right) .
\end{aligned}
$$

Equation (31) yields

$$
\sum_{n=0}^{\infty} F_{n}=A+B y+L^{-1}(G)-\widetilde{\beta} L^{-1}\left(\sum_{n=0}^{\infty} A_{n}+\sum_{n=0}^{\infty} B_{n}\right) .
$$

The associated boundary conditions (18) will be

$$
\begin{array}{ll}
\sum_{n=0}^{\infty} F_{n}=0, & y=0, \\
\sum_{n=0}^{\infty} F_{n}=V_{0}, & y=1 .
\end{array}
$$

The recursive relation in (33) and (34) gives the component problems

$$
F_{0}=A+B y+L^{-1}(G),
$$

along with boundary conditions

$$
\begin{gathered}
F_{0}=0, \quad y=0, \\
F_{0}=V_{0}, \quad y=1, \\
F_{j+1}=-\widetilde{\beta} L^{-1}\left(A_{j}+B_{j}\right), \quad j \geq 0
\end{gathered}
$$

together with the boundary conditions

$$
\begin{array}{ll}
\sum_{n=1}^{\infty} F_{n}=0, & y=0, \\
\sum_{n=1}^{\infty} F_{n}=0, & y=1 .
\end{array}
$$

The ADM solution to (33) along with the boundary conditions (34) will be

$$
F=\sum_{n=0}^{\infty} F_{n}
$$

5.1. Zeroth Component Solution. The relations (35) and (36) give the zeroth component problem

$$
F_{0}=A+B y+L^{-1}(G) \text {, }
$$

and the boundary conditions are

$$
\begin{gathered}
F_{0}=0 \quad \text { at } y=0, \\
F_{0}=V_{0} \quad \text { at } y=1,
\end{gathered}
$$

which gives the solution

$$
\begin{gathered}
u_{0}=\frac{U}{W} y+\frac{1}{2} P_{, x}\left(y^{2}-y\right), \\
w_{0}=y+\frac{1}{2} P_{, z}\left(y^{2}-y\right),
\end{gathered}
$$

which are the linearly viscous solutions to the problem.

5.2. First Component Solution. Equations (37) and (38) give

$$
\begin{gathered}
F_{1}=-\widetilde{\beta} L^{-1}\left(A_{0}+B_{0}\right), \\
F_{1}=0 \quad \text { at } y=0, \\
F_{1}=0 \quad \text { at } y=1,
\end{gathered}
$$

where the remainder term $R$ of the linear part is zero and

$$
\begin{gathered}
A_{0}=\left(\frac{d F_{0}}{d y}\right)^{2} \frac{d^{2} \bar{F}_{0}}{d y^{2}}, \\
B_{0}=2\left(\frac{d F_{0}}{d y} \frac{d^{2} F_{0}}{d y^{2}} \frac{d \bar{F}_{0}}{d y}\right)
\end{gathered}
$$

are Adomian polynomials. Using (45)-(46) in (44) results in

$$
\begin{aligned}
& u_{1}=-\tilde{\beta}\left\{L_{11}\left(y^{2}-y\right)+L_{12}\left(y^{3}-y\right)+L_{13}\left(y^{4}-y\right)\right\}, \\
& w_{1}=-\tilde{\beta}\left\{T_{11}\left(y^{2}-y\right)+T_{12}\left(y^{3}-y\right)+T_{13}\left(y^{4}-y\right)\right\},
\end{aligned}
$$

where $L_{11}, L_{12}, L_{13}, T_{11}, T_{12}$, and $T_{13}$ are constants given in appendix. 
5.3. Second Component Solution. The relations (37) and (38) give

$$
\begin{gathered}
F_{2}=-\widetilde{\beta} L^{-1}\left(A_{1}+B_{1}\right), \\
F_{2}=0 \text { at } y=0, \\
F_{2}=0 \text { at } y=h, \\
A_{1}=\left(\frac{d F_{0}}{d y}\right)^{2} \frac{d^{2} \bar{F}_{1}}{d y^{2}}+2 \frac{d F_{0}}{d y} \frac{d F_{1}}{d y} \frac{d^{2} \bar{F}_{0}}{d y^{2}} \\
B_{1}=2\left(\frac{d F_{0}}{d y} \frac{d^{2} F_{0}}{d y^{2}} \frac{d \bar{F}_{1}}{d y}+\frac{d F_{0}}{d y} \frac{d^{2} F_{1}}{d y^{2}} \frac{d \bar{F}_{0}}{d y}+\frac{d F_{1}}{d y} \frac{d^{2} F_{0}}{d y^{2}} \frac{d \bar{F}_{0}}{d y}\right),
\end{gathered}
$$

where $A_{1}$ and $B_{1}$ are Adomian polynomials.

Using (50) in (49), we get

$$
\begin{aligned}
u_{2}=\widetilde{\beta}^{2}\{ & L_{14}\left(y^{2}-y\right)+L_{15}\left(y^{3}-y\right)+L_{16}\left(y^{4}-y\right) \\
& \left.+L_{17}\left(y^{5}-y\right)+L_{18}\left(y^{6}-y\right)\right\}, \\
w_{2}=\widetilde{\beta}^{2}\{ & T_{14}\left(y^{2}-y\right)+T_{15}\left(y^{3}-y\right)+T_{16}\left(y^{4}-y\right) \\
& \left.+T_{17}\left(y^{5}-y\right)+T_{18}\left(y^{6}-y\right)\right\},
\end{aligned}
$$

where $L_{14}, L_{15}, L_{16}, L_{17}, L_{18}, T_{14}, T_{15}, T_{16}, T_{17}$, and $T_{18}$ are constants mentioned in appendix.

\subsection{Velocity Profiles}

5.4.1. Velocity Profile in $x$-Direction. Equations (42), (47), and (51) give the ADM solution for the velocity profile in the transverse plane

$$
\begin{aligned}
u= & \frac{U}{W} y+\left(\frac{1}{2} P_{, x}+\widetilde{\beta} L_{11}+\widetilde{\beta}^{2} L_{14}\right)\left(y^{2}-y\right) \\
& +\left(\widetilde{\beta} L_{12}+\widetilde{\beta}^{2} L_{15}\right)\left(y^{3}-y\right)+\left(\widetilde{\beta} L_{13}+\widetilde{\beta}^{2} L_{16}\right)\left(y^{4}-y\right) \\
& +\widetilde{\beta}^{2} L_{17}\left(y^{5}-y\right)+\widetilde{\beta}^{2} L_{18}\left(y^{6}-y\right) .
\end{aligned}
$$

5.4.2. Velocity Profile in z-Direction. Equations (43), (48) and (52) give the ADM solution for the velocity profile in the down channel direction

$$
\begin{aligned}
w= & y+\left(\frac{1}{2} P_{, z}+\widetilde{\beta} T_{11}+\widetilde{\beta}^{2} T_{14}\right)\left(y^{2}-y\right) \\
& +\left(\widetilde{\beta} T_{12}+\widetilde{\beta}^{2} T_{15}\right)\left(y^{3}-y\right)+\left(\widetilde{\beta} T_{13}+\widetilde{\beta}^{2} T_{16}\right)\left(y^{4}-y\right) \\
& +\widetilde{\beta}^{2} T_{17}\left(y^{5}-y\right)+\widetilde{\beta}^{2} T_{18}\left(y^{6}-y\right) .
\end{aligned}
$$

5.4.3. Velocity in the Direction of the Axis of Screw. The velocity in the direction of the axis of the screw at any depth in the channel can be computed from (53) and (54) as

$$
\begin{aligned}
s=w \sin \phi+u \cos \phi, \\
s=\left\{y+\left(\frac{1}{2} P_{, z}+\widetilde{\beta} T_{11}+\widetilde{\beta}^{2} T_{14}\right)\left(y^{2}-y\right)\right. \\
+\left(\widetilde{\beta} T_{12}+\widetilde{\beta}^{2} T_{15}\right)\left(y^{3}-y\right) \\
+\left(\widetilde{\beta} T_{13}+\widetilde{\beta}^{2} T_{16}\right)\left(y^{4}-y\right)+\widetilde{\beta}^{2} T_{17}\left(y^{5}-y\right) \\
\left.+\widetilde{\beta}^{2} T_{18}\left(y^{6}-y\right)\right\} \sin \phi \\
+\left\{\frac{U}{W} y+\left(\frac{1}{2} P_{, x}+\widetilde{\beta} L_{11}+\widetilde{\beta}^{2} L_{14}\right)\left(y^{2}-y\right)\right. \\
+\left(\widetilde{\beta} L_{12}+\widetilde{\beta}^{2} L_{15}\right)\left(y^{3}-y\right) \\
+\left(\widetilde{\beta} L_{13}+\widetilde{\beta}^{2} L_{16}\right)\left(y^{4}-y\right)+\widetilde{\beta}^{2} L_{17}\left(y^{5}-y\right) \\
\left.+\widetilde{\beta}^{2} L_{18}\left(y^{6}-y\right)\right\} \cos \phi,
\end{aligned}
$$

which shows the resultant velocity of the flow.

5.5. Volume Flow Rates. Volume flow rate in $x$-direction per unit width is

$$
Q_{x}^{*}=\int_{0}^{1} u d y
$$

where $Q_{x}^{*}=Q_{x} / W h B$, and (57) gives

$$
\begin{aligned}
Q_{x}^{*}= & \frac{U}{2 W}-\frac{1}{6}\left(\frac{1}{2} P_{, x}+\widetilde{\beta} L_{11}+\widetilde{\beta}^{2} L_{14}\right)-\frac{1}{4}\left(\widetilde{\beta} L_{12}+\widetilde{\beta}^{2} L_{15}\right) \\
& -\frac{3}{10}\left(\widetilde{\beta} L_{13}+\widetilde{\beta}^{2} L_{16}\right)-\frac{1}{3} \widetilde{\beta}^{2} L_{17}-\frac{5}{14} \widetilde{\beta}^{2} L_{18} .
\end{aligned}
$$

Volume flow rate in $z$-direction per unit width is

$$
Q_{z}^{*}=\int_{0}^{1} w d y,
$$

where $Q_{z}^{*}=Q_{z} / W h B$, and (59) gives

$$
\begin{aligned}
Q_{z}^{*}= & \frac{1}{2}-\frac{1}{6}\left(\frac{1}{2} P_{, z}+\widetilde{\beta} T_{11}+\widetilde{\beta}^{2} T_{14}\right)-\frac{1}{4}\left(\widetilde{\beta} T_{12}+\widetilde{\beta}^{2} T_{15}\right) \\
& -\frac{3}{10}\left(\widetilde{\beta} T_{13}+\widetilde{\beta}^{2} T_{16}\right)-\frac{1}{3} \widetilde{\beta}^{2} T_{17}-\frac{5}{14} \widetilde{\beta}^{2} T_{18} .
\end{aligned}
$$

Equation (56) gives the resultant volume flow rate forward in the screw channel, which is the product of the velocity and cross-sectional area integrated from the root of the screw to the barrel surface

$$
Q^{*}=\frac{n}{\sin \phi} \int_{0}^{1} s d y
$$




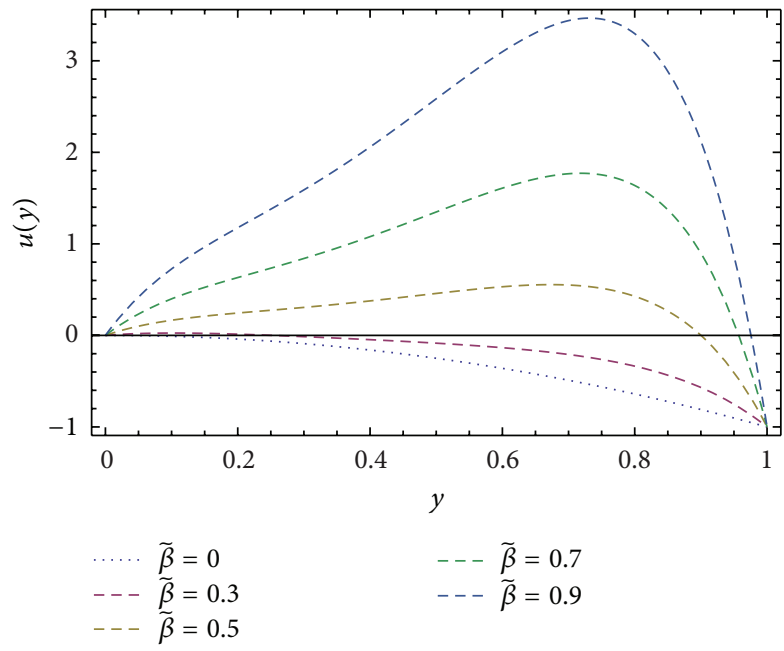

Figure 2: Profile of the nondimensional velocity $u(y)$ for different values of $\widetilde{\beta}$, keeping $P_{, x}=-2.0, P_{, z}=-2.0$, and $\phi=45^{\circ}$.

where $Q^{*}=Q / W h B$ and $n$ is the number of parallel flights in a multiflight screw.

Equation (61) gives

$$
\begin{aligned}
Q^{*}=\frac{n}{\sin \phi}[ & \left\{\frac{1}{2}-\frac{1}{6}\left(\frac{1}{2} P_{, z}+\widetilde{\beta} T_{11}+\widetilde{\beta}^{2} T_{14}\right)\right. \\
& -\frac{1}{4}\left(\widetilde{\beta} T_{12}+\widetilde{\beta}^{2} T_{15}\right)-\frac{3}{10}\left(\widetilde{\beta} T_{13}+\widetilde{\beta}^{2} T_{16}\right) \\
& \left.-\frac{1}{3} \widetilde{\beta}^{2} T_{17}-\frac{5}{14} \widetilde{\beta}^{2} T_{18}\right\} \sin \phi \\
+ & \left\{\frac{U}{2 W}-\frac{1}{6}\left(\frac{1}{2} P_{, x}+\widetilde{\beta} L_{11}+\widetilde{\beta}^{2} L_{14}\right)\right. \\
& \quad-\frac{1}{4}\left(\widetilde{\beta} L_{12}+\widetilde{\beta}^{2} L_{15}\right)-\frac{3}{10}\left(\widetilde{\beta} L_{13}+\widetilde{\beta}^{2} L_{16}\right) \\
& \left.\left.\quad-\frac{1}{3} \widetilde{\beta}^{2} L_{17}-\frac{5}{14} \widetilde{\beta}^{2} L_{18}\right\} \cos \phi\right],
\end{aligned}
$$

which can be written as

$$
Q^{*}=\frac{n}{\sin \phi}\left\{Q_{z}^{*} \sin \phi+Q_{x}^{*} \cos \phi\right\} .
$$

\section{Results and Discussion}

In the present work, we have considered the steady flow of an incompressible, isothermal, and homogeneous thirdgrade fluid in helical screw rheometer (HSR). Using Adomian decomposition method, solutions are obtained for velocity profiles in $x$-, $z$-directions and also in the direction of the axis of the screw $s(y)$. The volume flow rates are also calculated by using the velocities in $x, z$ and in the direction of the axis of the screw. Here we discussed the effect of dimensionless parameters $\widetilde{\beta}, U / W=-\tan \phi, P_{, x}$, and $P_{, z}$ where $\phi=45^{\circ}$, on the velocity profiles given in (53), (54), and (56) with the

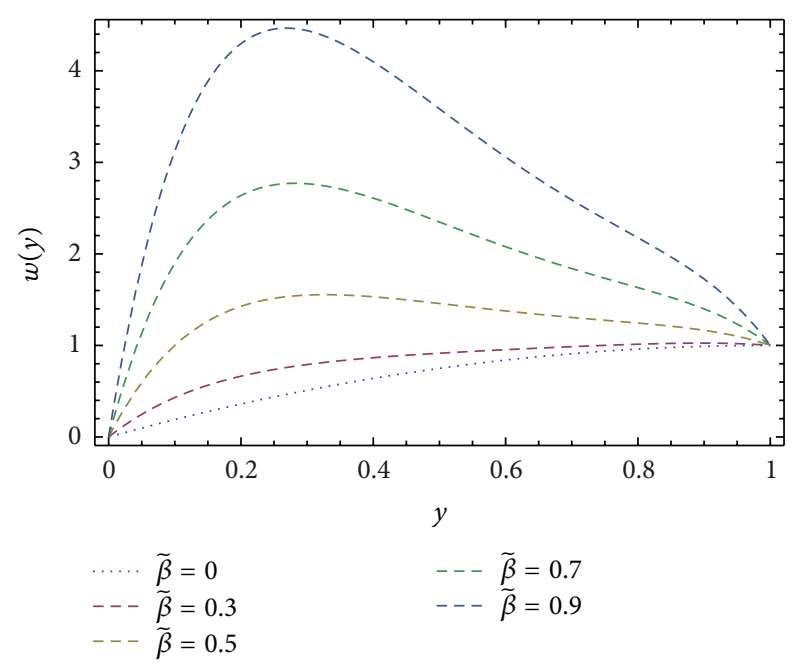

Figure 3: Profile of the nondimensional velocity $w(y)$ for different values of $\widetilde{\beta}$, keeping $P_{, x}=-2.0, P_{, z}=-2.0$, and $\phi=45^{\circ}$.

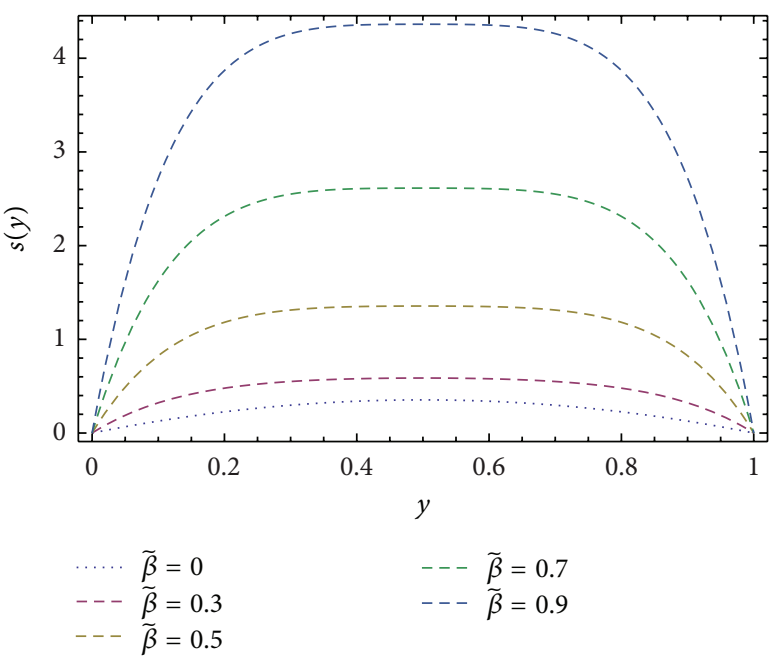

Figure 4: Profile of the nondimensional velocity $s(y)$ for different values of $\widetilde{\beta}$, keeping $P_{, x}=-2.0, P_{, z}=-2.0$, and $\phi=45^{\circ}$.

help of graphical representation. Figures 2, 3, and 4 for the velocities in $x$-direction $u(y), z$-direction $w(y)$, and the resultant velocity $s(y)$ are plotted against $y$ for different values of non-Newtonian parameter $\widetilde{\beta}$ and constant pressure gradients $P_{, x}=-2.0, P_{, z}=-2.0$, respectively. From these figures, it is seen that the velocity profiles are strongly dependant on the non-Newtonian parameter $\widetilde{\beta}$, as we increase the value of $\widetilde{\beta}$ in the interval 0 to 0.9 , the progressive increase in velocities in $x$-, $z$-direction and in the direction of the axis of screw found. It is worthwhile to note that the extrusion process increases with the increase of the non-Newtonian parameter $\widetilde{\beta}$.

Figures 5, 7, and 9 are sketched for the velocity profiles $u(y), w(y)$, and $s(y)$ against $y$ for different values of $P_{, x}$, keeping $\widetilde{\beta}=0.3$ and $P_{, z}=-2.0$ fixed; enlightened escalation 


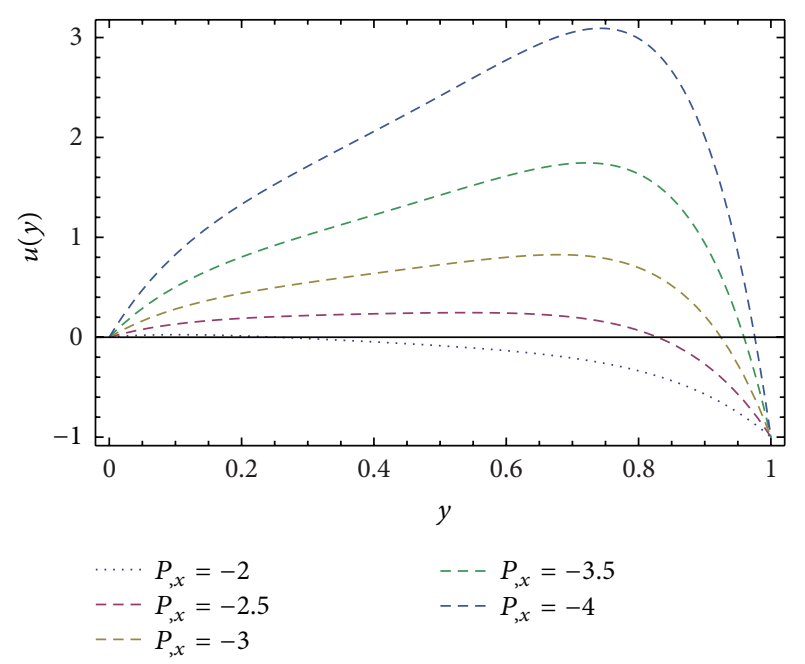

FIGURE 5: Profile of the nondimensional velocity $u(y)$ for different values of $P_{, x}$, keeping $\widetilde{\beta}=0.3, P_{, z}=-2.0$, and $\phi=45^{\circ}$.

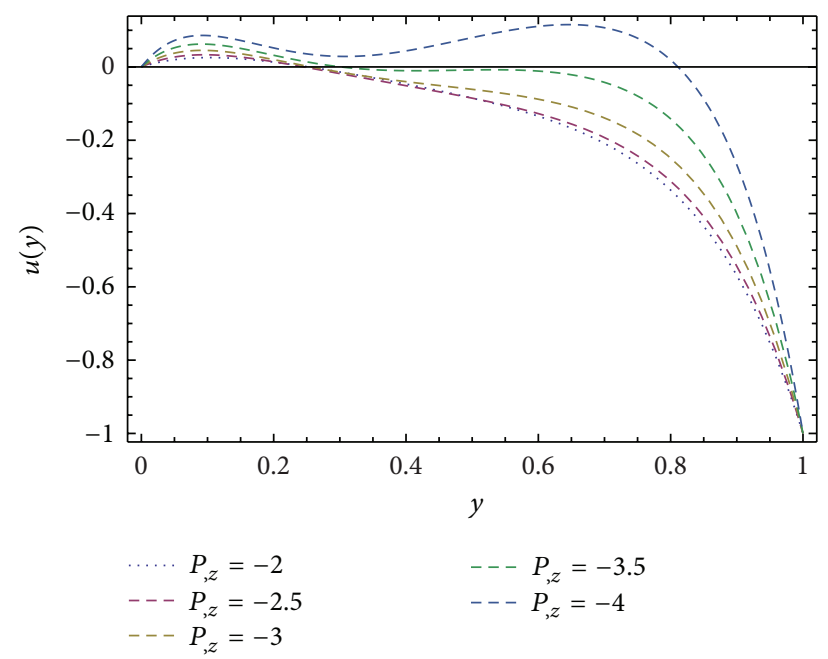

Figure 6: Profile of the nondimensional velocity $u(y)$ for different values of $P_{, z}$, keeping $\widetilde{\beta}=0.3, P_{, x}=-2.0$, and $\phi=45^{\circ}$.

is noted in the velocity profiles with increase in pressure gradient in $x$-direction.

Figures 6,8 , and 10 are sketched for the velocity profiles $u(y), w(y)$, and $s(y)$ against $y$ for different values of $P_{, z}$, keeping $\widetilde{\beta}=0.3$ and $P_{, x}=-2.0$ fixed, it is observed that parabolicity of the velocity profiles increases with increase in pressure gradient in $z$-direction.

Figure 11 is plotted for the velocity $s(y)$ against $y$ for different values of $\phi$, keeping $\widetilde{\beta}=0.3, P_{, x}=-2.0$, and $P_{, z}=-2.0$. It is observed that flow increases as the flight angle increases up to $\phi=45^{\circ}$.

\section{Conclusion}

The steady flow of an isothermal, homogeneous and incompressible third-grade fluid is investigated in helical screw

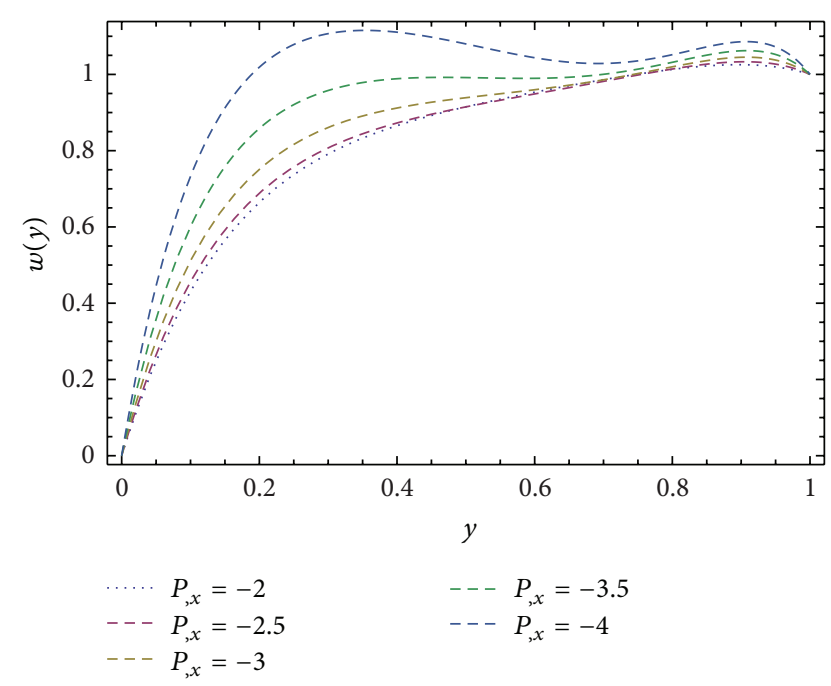

Figure 7: Profile of the nondimensional velocity $w(y)$ for different values of $P_{, x}$, keeping $\widetilde{\beta}=0.3, P_{, z}=-2.0$, and $\phi=45^{\circ}$.

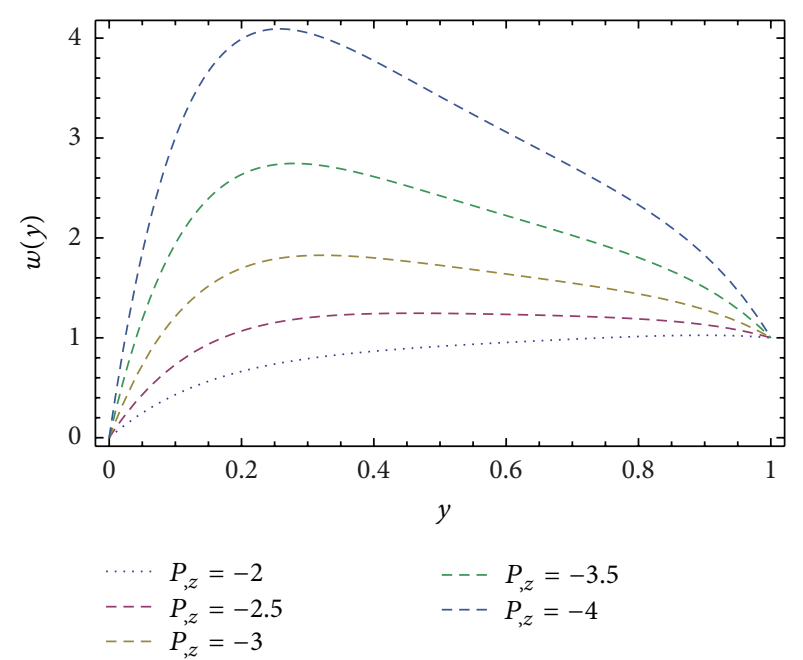

FIGURE 8: Profile of the nondimensional velocity $w(y)$ for different values of $P_{, z}$, keeping $\widetilde{\beta}=0.3, P_{, x}=-2.0$, and $\phi=45^{\circ}$.

rheometer (HSR). The geometry of the problem under consideration gives second-order nonlinear coupled differential equations which are reduced to single differential equation by using a transformation. Adomian decomposition method is used to obtain analytical expressions for the flow profiles, volume flow rate. It is noticed that the zeroth component solution matches with solution of the linearly viscous fluid in HSR, and it is also found that the net velocity of the fluid is due to the pressure gradient as the expression for the net velocity is free from the drag term. Graphical representation shows that the velocity profiles are strongly dependant on non-Newtonian parameter $(\widetilde{\beta})$ and pressure gradients in $x$ and $z$-direction. Thus, the extrusion process strongly depends on the involved parameters. 


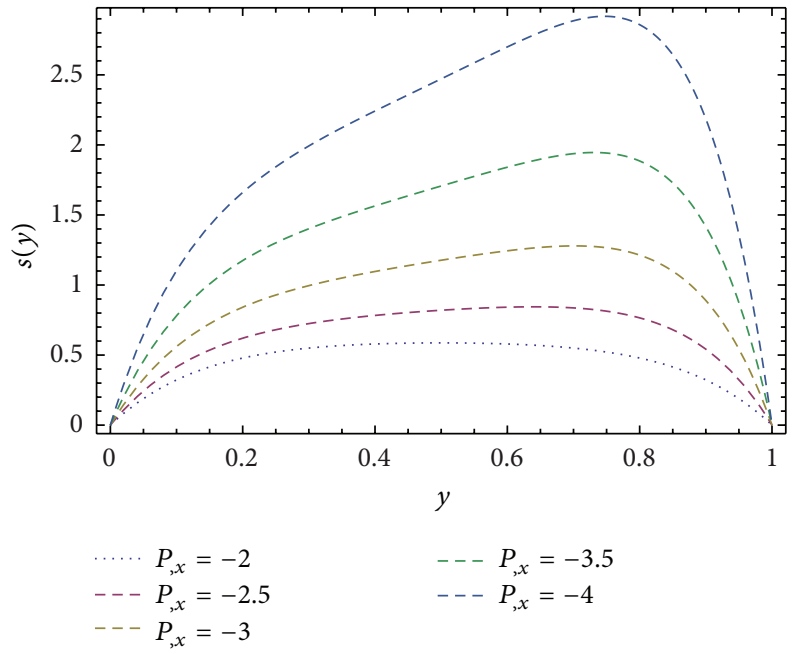

Figure 9: Profile of the nondimensional velocity $s(y)$ for different values of $P_{, x}$, keeping $\widetilde{\beta}=0.3, P_{, z}=-2.0$, and $\phi=45^{\circ}$.

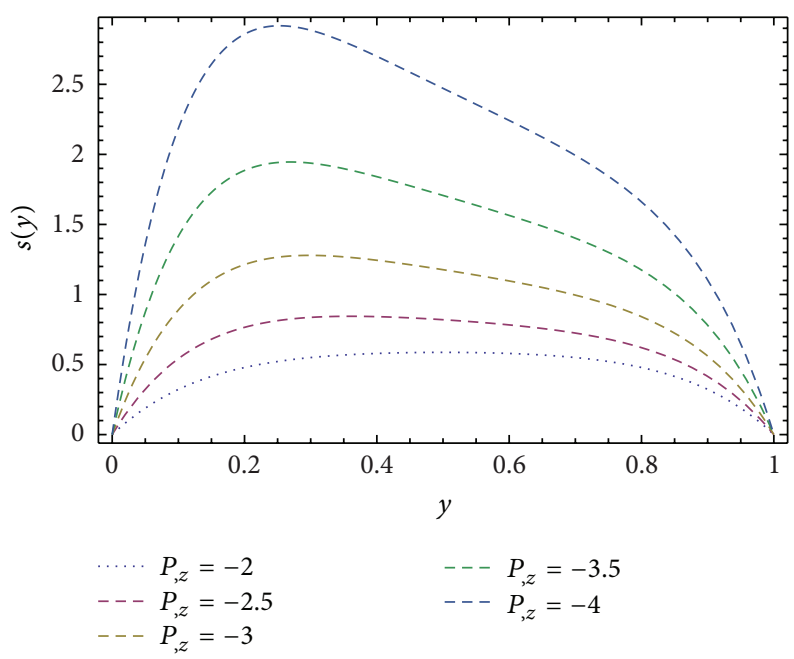

Figure 10: Profile of the nondimensional velocity $s(y)$ for different values of $P_{, z}$, keeping $\widetilde{\beta}=0.3, P_{, x}=-2.0$, and $\phi=45^{\circ}$.

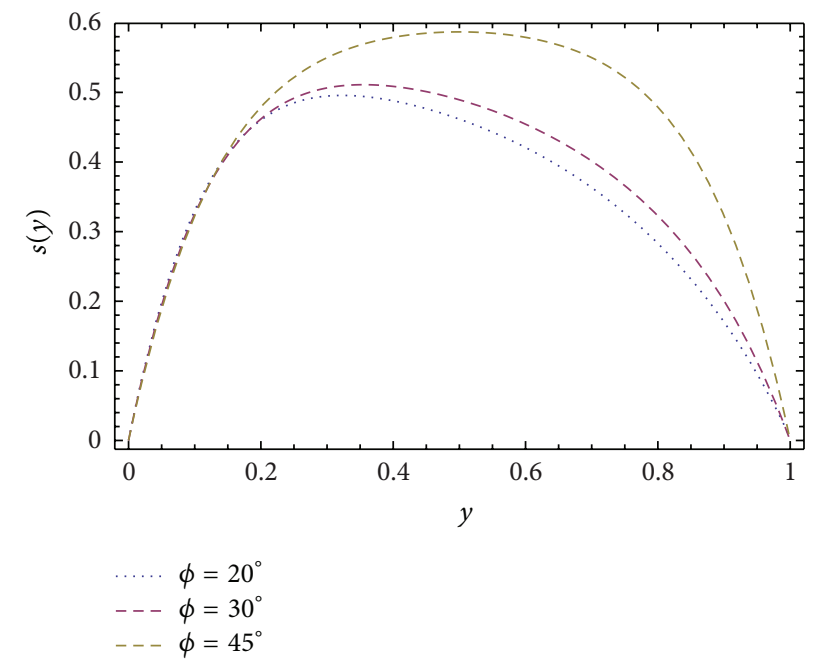

FIGURE 11: Profile of the nondimensional velocity $s(y)$ for different values of $P_{, z}$, keeping $\widetilde{\beta}=0.3, P_{, x}=-2.0$, and $\phi=45^{\circ}$.

\section{Appendix}

$$
\begin{aligned}
& L_{11}=\frac{1}{2}\left(P_{1}+\frac{3 U^{2} P_{1}}{W^{2}}-\frac{3 U P_{1}^{2}}{W}+\frac{3 P_{1}^{3}}{4}\right. \\
& \left.+\frac{2 U P_{2}}{W}-2 P_{1} P_{2}-\frac{U P_{2}^{2}}{W}+\frac{3}{4} P_{1} P_{2}^{2}\right), \\
& L_{12}=\frac{1}{6}\left(\frac{6 U P_{1}^{2}}{W}-3 P_{1}^{3}+4 P_{1} P_{2}+\frac{2 U P_{2}^{2}}{W}-3 P_{1} P_{2}^{2}\right) \text {, } \\
& L_{13}=\frac{1}{12}\left(3 P_{1}^{3}+3 P_{1} P_{2}^{2}\right), \\
& L_{14}=\frac{1}{2}\left(P_{1}+\frac{9 U^{4} P_{1}}{W^{4}}+\frac{10 U^{2} P_{1}}{W^{2}}-\frac{27 U^{3} P_{1}^{2}}{W^{3}}\right. \\
& -\frac{15 U P_{1}^{2}}{W}+\frac{13 P_{1}^{3}}{3}+\frac{21 U^{2} P_{1}^{3}}{W^{2}} \\
& -\frac{27 U P_{1}^{4}}{4 W}+\frac{15 P_{1}^{5}}{16}+\frac{8 U^{3} P_{2}}{W^{3}}+\frac{8 U P_{2}}{W} \\
& -10 P_{1} P_{2}-\frac{34 U^{2} P_{1} P_{2}}{W^{2}}+\frac{25 U P_{1}^{2} P_{2}}{W} \\
& -\frac{16}{3} P_{1}^{3} P_{2}-\frac{5 U^{3} P_{2}^{2}}{W^{3}}-\frac{17 U P_{2}^{2}}{W}+\frac{38}{3} P_{1} P_{2}^{2} \\
& +\frac{38 U^{2} P_{1} P_{2}^{2}}{3 W^{2}}-\frac{49 U P_{1}^{2} P_{2}^{2}}{6 W}+\frac{15}{8} P_{1}^{3} P_{2}^{2} \\
& \left.+\frac{25 U P_{2}^{3}}{3 W}-\frac{16}{3} P_{1} P_{2}^{3}-\frac{17 U P_{2}^{4}}{12 W}+\frac{15}{16} P_{1} P_{2}^{4}\right), \\
& L_{15}=\frac{1}{6}\left(\frac{54 U^{3} P_{1}^{2}}{W^{3}}+\frac{30 U P_{1}^{2}}{W}-18 P_{1}^{3}\right. \\
& -\frac{90 U^{2} P_{1}^{3}}{W^{2}}+\frac{87 U P_{1}^{4}}{2 W}-\frac{15 P_{1}^{5}}{2} \\
& +\frac{68 U^{2} P_{1} P_{2}}{W^{2}}-\frac{108 U P_{1}^{2} P_{2}}{W}+\frac{104}{3} P_{1}^{3} P_{2} \\
& +\frac{10 U^{3} P_{2}^{2}}{W^{3}}+\frac{34 U P_{2}^{2}}{W}-54 P_{1} P_{2}^{2} \\
& -\frac{54 U^{2} P_{1} P_{2}^{2}}{W^{2}}+\frac{157 U P_{1}^{2} P_{2}^{2}}{3 W}-15 P_{1}^{3} P_{2}^{2}-\frac{36 U P_{2}^{3}}{W} \\
& \left.+\frac{104}{3} P_{1} P_{2}^{3}+\frac{53 U P_{2}^{4}}{6 W}-\frac{15}{2} P_{1} P_{2}^{4}+20 P_{1} P_{2}\right), \\
& L_{16}=\frac{1}{12}\left(18 P_{1}^{3}+\frac{90 U^{2} P_{1}^{3}}{W^{2}}-\frac{90 U P_{1}^{4}}{W}+\frac{45 P_{1}^{5}}{2}\right. \\
& +\frac{108 U P_{1}^{2} P_{2}}{W}-72 P_{1}^{3} P_{2}+54 P_{1} P_{2}^{2}
\end{aligned}
$$




$$
\begin{aligned}
& +\frac{54 U^{2} P_{1} P_{2}^{2}}{W^{2}}-\frac{108 U P_{1}^{2} P_{2}^{2}}{W}+45 P_{1}^{3} P_{2}^{2}+\frac{36 U P_{2}^{3}}{W} \\
& \left.-72 P_{1} P_{2}^{3}-\frac{18 U P_{2}^{4}}{W}+\frac{45}{2} P_{1} P_{2}^{4}\right), \\
& L_{17}=\frac{1}{20}\left(\frac{60 U P_{1}^{4}}{W}-30 P_{1}^{5}+48 P_{1}^{3} P_{2}+\frac{72 U P_{1}^{2} P_{2}^{2}}{W}-60 P_{1}^{3} P_{2}^{2}\right. \\
& \left.+48 P_{1} P_{2}^{3}+\frac{12 U P_{2}^{4}}{W}-30 P_{1} P_{2}^{4}\right) \\
& L_{18}=\frac{1}{30}\left(15 P_{1}^{5}+30 P_{1}^{3} P_{2}^{2}+15 P_{1} P_{2}^{4}\right) \text {, } \\
& T_{11}=\frac{1}{2}\left(2 \frac{U P_{1}}{W}-P_{1}^{2}+3 P_{2}+\frac{U^{2} P_{2}}{W^{2}}\right. \\
& \left.-2 \frac{U P_{1} P_{2}}{W}+\frac{3}{4} P_{1}^{2} P_{2}-3 P_{2}^{2}+\frac{3}{4} P_{2}^{3}\right), \\
& T_{12}=\frac{1}{6}\left(2 P_{1}^{2}+4 \frac{U P_{1} P_{2}}{W}-3 P_{1}^{2} P_{2}+6 P_{2}^{2}-3 P_{2}^{3}\right), \\
& T_{13}=\frac{1}{12}\left(3 P_{1}^{2} P_{2}+3 P_{2}^{3}\right), \\
& T_{14}=\frac{1}{2}\left(\frac{8 U^{3} P_{1}}{W^{3}}+\frac{8 U P_{1}}{W}-5 P_{1}^{2}-\frac{17 U^{2} P_{1}^{2}}{W^{2}}\right. \\
& +\frac{25 U P_{1}^{3}}{3 W}-\frac{17 P_{1}^{4}}{12}+9 P_{2}+\frac{U^{4} P_{2}}{W^{4}} \\
& +\frac{10 U^{2} P_{2}}{W^{2}}-\frac{10 U^{3} P_{1} P_{2}}{W^{3}}-\frac{34 U P_{1} P_{2}}{W} \\
& +\frac{38}{3} P_{1}^{2} P_{2}+\frac{38 U^{2} P_{1}^{2} P_{2}}{3 W^{2}}-\frac{16 U P_{1}^{3} P_{2}}{3 W} \\
& +\frac{15}{16} P_{1}^{4} P_{2}-27 P_{2}^{2}-\frac{15 U^{2} P_{2}^{2}}{W^{2}}+\frac{25 U P_{1} P_{2}^{2}}{W} \\
& -\frac{49}{6} P_{1}^{2} P_{2}^{2}+21 P_{2}^{3}+\frac{13 U^{2} P_{2}^{3}}{3 W^{2}} \\
& \left.-\frac{16 U P_{1} P_{2}^{3}}{3 W}+\frac{15}{8} P_{1}^{2} P_{2}^{3}-\frac{27 P_{2}^{4}}{4}+\frac{15 P_{2}^{5}}{16}\right), \\
& T_{15}=\frac{1}{6}\left(10 P_{1}^{2}+\frac{34 U^{2} P_{1}^{2}}{W^{2}}-\frac{36 U P_{1}^{3}}{W}+\frac{53 P_{1}^{4}}{6}\right. \\
& +\frac{20 U^{3} P_{1} P_{2}}{W^{3}}+\frac{68 U P_{1} P_{2}}{W}-54 P_{1}^{2} P_{2} \\
& -\frac{54 U^{2} P_{1}^{2} P_{2}}{W^{2}}+\frac{104 U P_{1}^{3} P_{2}}{3 W}-\frac{15}{2} P_{1}^{4} P_{2} \\
& +54 P_{2}^{2}+\frac{30 U^{2} P_{2}^{2}}{W^{2}}-\frac{108 U P_{1} P_{2}^{2}}{W}
\end{aligned}
$$

$$
\begin{gathered}
+\frac{157}{3} P_{1}^{2} P_{2}^{2}-90 P_{2}^{3}-\frac{18 U^{2} P_{2}^{3}}{W^{2}} \\
\left.+\frac{104 U P_{1} P_{2}^{3}}{3 W}-15 P_{1}^{2} P_{2}^{3}+\frac{87 P_{2}^{4}}{2}-\frac{15 P_{2}^{5}}{2}\right) \\
T_{16}=\frac{1}{12}\left(\frac{36 U P_{1}^{3}}{W}-18 P_{1}^{4}+54 P_{1}^{2} P_{2}+\frac{54 U^{2} P_{1}^{2} P_{2}}{W^{2}}\right. \\
\quad-\frac{72 U P_{1}^{3} P_{2}}{W}+\frac{45}{2} P_{1}^{4} P_{2} \\
+\frac{108 U P_{1} P_{2}^{2}}{W}-108 P_{1}^{2} P_{2}^{2}+90 P_{2}^{3}+\frac{18 U^{2} P_{2}^{3}}{W^{2}} \\
\left.\quad-\frac{72 U P_{1} P_{2}^{3}}{W}+45 P_{1}^{2} P_{2}^{3}-90 P_{2}^{4}+\frac{45 P_{2}^{5}}{2}\right) \\
T_{17}=\frac{1}{20}\left(\begin{array}{ll}
12 P_{1}^{4}+\frac{48 U P_{1}^{3} P_{2}}{W}-30 P_{1}^{4} P_{2}+72 P_{1}^{2} P_{2}^{2} \\
W
\end{array}\right. \\
\left.+\frac{1}{30}\left(15 P_{1}^{4} P_{2}+30 P_{1}^{2} P_{2}^{3}+15 P_{2}^{5}\right) \cdot 6 P_{1}^{2} P_{2}^{3}+60 P_{2}^{4}-30 P_{2}^{5}\right)
\end{gathered}
$$

\section{Acknowledgment}

The first author is very thankful to Higher Education Commission (HEC) of Pakistan for funding his higher studies under the 5000 indigenous scholarship scheme Batch-IV.

\section{References}

[1] R. S. Rivlin and I. L. Erickson, "Stress deformation relations for isotropic materials," Journal of Rational Mechanics and Analysis, vol. 4, pp. 323-425, 1955.

[2] M. Alinia, D. D. Ganji, and M. Gorji-Bandpy, "Numerical study of mixed convection in an inclined two sided lid driven cavity filled with nanofluid using two-phase mixture model," International Communications in Heat and Mass Transfer, vol. 38, no. 10, pp. 1428-1435, 2011.

[3] M. Sheikholeslami, M. Gorji-Bandpy, D. D. Ganji, S. Soleimani, and S. M. Seyyedi, "Natural convection of nanofluids in an enclosure between a circular and a sinusoidal cylinder in the presence of magnetic field," International Communications in Heat and Mass Transfer, vol. 39, no. 9, pp. 1435-1443, 2012.

[4] M. Sheikholeslami, M. Gorji-Bandpy, and D. D. Ganji, "Magnetic field effects on natural convection around a horizontal circular cylinder inside a square enclosure filled with nanofluid," International Communications in Heat and Mass Transfer, vol. 39, no. 7, pp. 978-986, 2012.

[5] D. D. Ganji, "A semi Analytical technique for non linear settling particle equation of Motion," Journal of Hydro Environment Research, vol. 6, no. 4, pp. 323-327, 2012. 
[6] M. Sheikholeslami and D. D. Ganji, "Heat transfer of Cu water nanofluid flow between parallel plates," Powder Technology, vol. 235, pp. 873-879, 2013.

[7] S. M. Hamidi, Y. Rostamiyan, D. D. Ganji, and A. Fereidoon, "A novel and developed approximation for motion of a spherical solid particle in plane coquette fluid flow," Advanced Poweder Tecnology, 2013.

[8] A. H. Nayfeh, Introduction to Perturbation Techniques, Wiley, New York, NY, USA, 1979.

[9] J. H. He, "Homotopy perturbation technique," Computer Methods in Applied Mechanics and Engineering, vol. 178, no. 3-4, pp. 257-262, 1999.

[10] M. Grover and A. K. Tomer, "Comparison of optimal homotopy asymptotic method with homotopy perturbation method of twelfth order boundary value problems," International Journal on Computer Science and Engineering, vol. 3, no. 7, pp. 27392747, 2011.

[11] J. H. He, "Asymptotology by homotopy perturbation method," Applied Mathematics and Computation, vol. 156, no. 3, pp. 591596, 2004.

[12] G. Adomian, Stochastic Systems, Academic Press, Orlando, Fla, USA, 1983.

[13] G. Adomian, Nonlinear Stochastic Operator Equations, Academic Press, Orlando, Fla, USA, 1986.

[14] A. M. Wazwaz, "A comparison between Adomian decomposition method and Taylor series method in the series solutions," Applied Mathematics and Computation, vol. 97, no. 1, pp. 37-44, 1998.

[15] A. M. Wazwaz, "Analytical solution for the time-dependent Emden-Fowler type of equations by Adomian decomposition method," Applied Mathematics and Computation, vol. 166, no. 3, pp. 638-651, 2005.

[16] A. M. Siddiqui, M. Hameed, B. M. Siddiqui, and Q. K. Ghori, "Use of Adomian decomposition method in the study of parallel plate flow of a third grade fluid," Communications in Nonlinear Science and Numerical Simulation, vol. 15, no. 9, pp. 2388-2399, 2010.

[17] A. M. Wazwaz, Partial Differential Equations Methods and Applications, A. A. Balkema, Tokyo, Japan, 2002.

[18] M. S. Tamura, J. M. Henderson, R. L. Powell, and C. F. Shoemaker, "Analysis of the helical screw rheometer for fluid food," Journal of Food Process Engineering, vol. 16, no. 2, pp. 93-126, 1993.

[19] A. M. Siddiqui, T. Haroon, and S. Irum, "Torsional flow of third grade fluid using modified homotopy perturbation method," Computers \& Mathematics with Applications, vol. 58, no. 11-12, pp. 2274-2285, 2009.

[20] R. V. Chiruvella, Y. Jaluria, V. Sernas, and M. Esseghir, "Extrusion of non-Newtonian fluids in a single-screw extruder with pressure back flow," Polymer Engineering and Science, vol. 36, no. 3, pp. 358-367, 1996.

[21] M. A. Rao, "Rheology of liquid foods: a review," Journal of Texture Studies, vol. 8, no. 2, pp. 135-168, 1977.

[22] P. J. Tily, "Viscosity measurement (part 2)," Measurement and Control, vol. 16, no. 4, pp. 137-139, 1983.

[23] R. B. Bird, R. C. Armstrong, and O. Hassager, "Enhancement of axial annular flow by rottaing inner cylinder," in Dynamics of Ploymeric Liquids, Fluid Mechanics, vol. 1, pp. 184-187, Wiley, New York, NY, USA, 1987.

[24] W. D. Mohr and R. S. Mallouk, "Power requirement and pressure distribution of fluid in a screw extruder," Industrial and Engineering Chemistry, vol. 51, no. 6, pp. 765-770, 1959.
[25] G. Adomian and R. Rach, "On the solution of algebraic equations by the decomposition method," Journal of Mathematical Analysis and Applications, vol. 105, no. 1, pp. 141-166, 1985.

[26] G. Adomian, "Convergent series solution of nonlinear equations," Journal of Computational and Applied Mathematics, vol. 11, no. 2, pp. 225-230, 1984.

[27] G. Adomian, "A review of the decomposition method in applied mathematics," Journal of Mathematical Analysis and Applications, vol. 135, no. 2, pp. 501-544, 1988. 


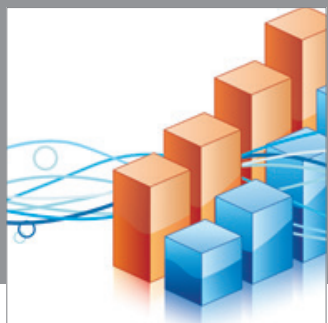

Advances in

Operations Research

mansans

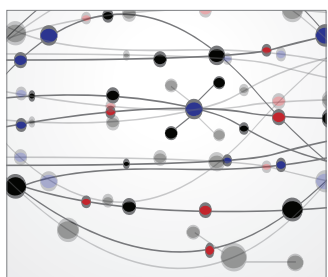

The Scientific World Journal
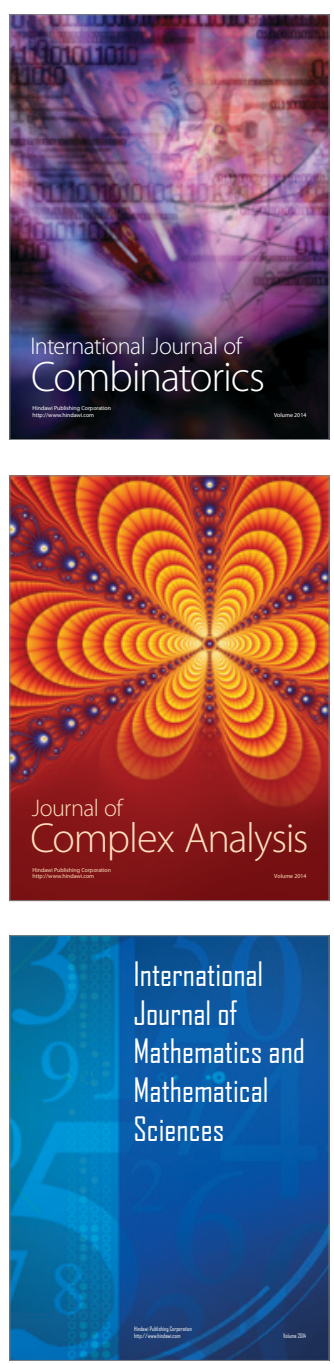
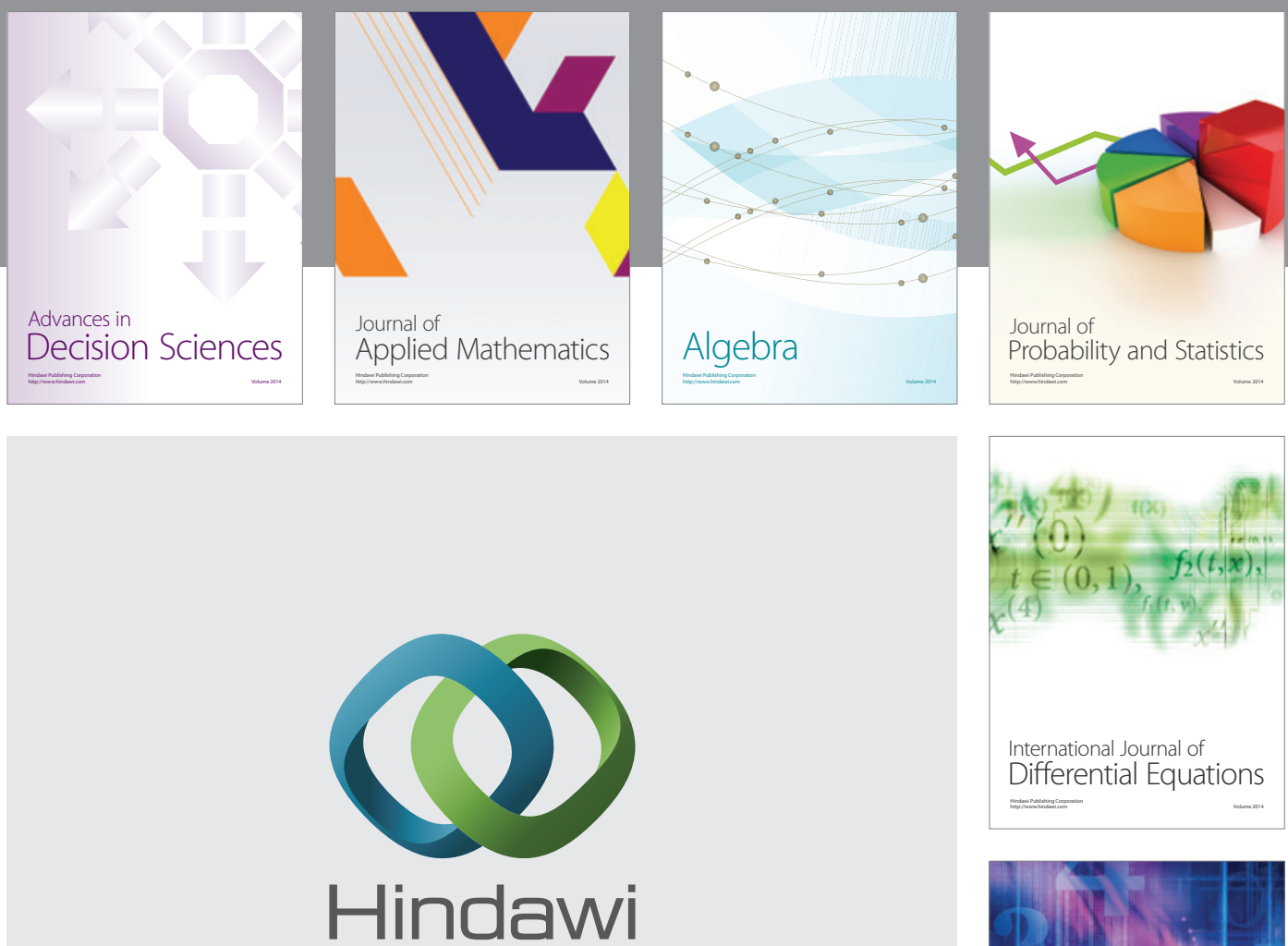

Submit your manuscripts at http://www.hindawi.com
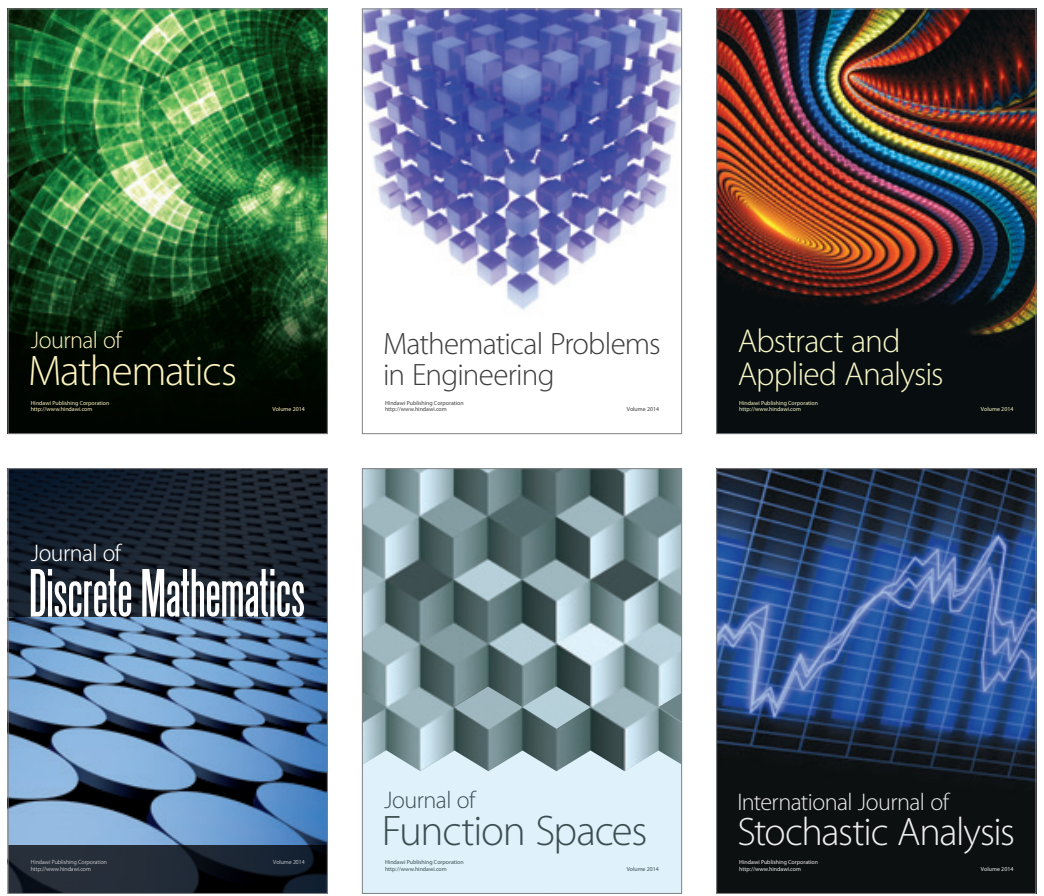

Journal of

Function Spaces

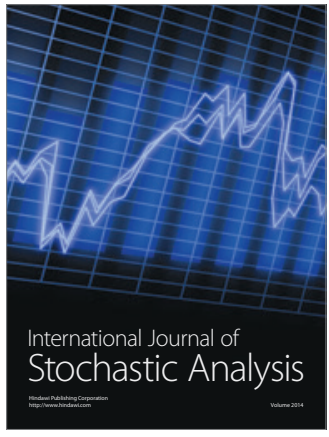

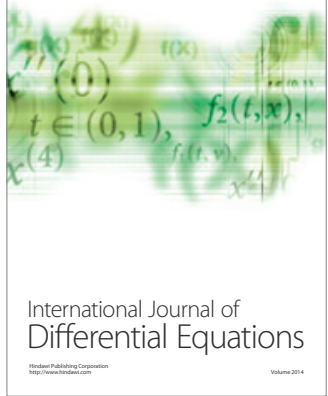
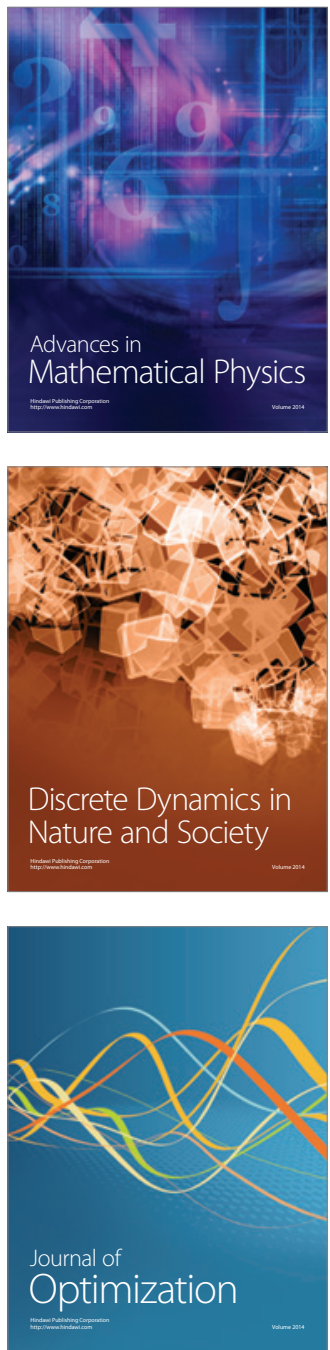